\title{
Scattering resonances and bound states for strongly interacting Rydberg polaritons
}

\author{
P. Bienias, ${ }^{1, *}, \dagger$ S. Choi, ${ }^{2,}{ }^{*}$ O. Firstenberg, ${ }^{2}$ M. F. Maghrebi,${ }^{3}$ M. Gullans, ${ }^{3}$ M. D. Lukin, ${ }^{2}$ A. V. Gorshkov, ${ }^{3}$ and H. P. Büchler ${ }^{1}$ \\ ${ }^{1}$ Institute for Theoretical Physics III, University of Stuttgart, 70550 Stuttgart, Germany \\ ${ }^{2}$ Department of Physics, Harvard University, Cambridge, Massachusetts 02138, USA \\ ${ }^{3}$ Joint Quantum Institute, National Institute of Standards and Technology, and University of Maryland, College Park, Maryland 20742, USA
}

(Received 1 March 2014; published 3 November 2014)

\begin{abstract}
We provide a theoretical framework describing slow-light polaritons interacting via atomic Rydberg states. The method allows us to analytically derive the scattering properties of two polaritons. We identify parameter regimes where polariton-polariton interactions are repulsive. Furthermore, in the regime of attractive interactions, we identify multiple two-polariton bound states, calculate their dispersion, and study the resulting scattering resonances. Finally, the two-particle scattering properties allow us to derive the effective low-energy many-body Hamiltonian. This theoretical platform is applicable to ongoing experiments.
\end{abstract}

DOI: 10.1103/PhysRevA.90.053804

PACS number(s): 42.50.Nn, 32.80.Ee, 34.20.Cf, 42.50.Gy

\section{INTRODUCTION}

Weak interactions of photons with each other are the basis for many applications of light signals in areas such as optical communication. However, many other applications in classical and quantum communication, computation, and metrology would greatly benefit from tunable photon-photon interactions. Moreover, photon-photon interactions at the level of individual quanta could pave the way for the realization of exotic strongly correlated photonic states [1-3]. A typical approach to achieve strong two-photon interactions relies on confining photons to high-finesse cavities [4-7]. An alternative approach towards this goal has recently emerged using Rydberg slow-light polaritons [8-15].

The key idea [16] is to combine electromagnetically induced transparency (EIT) $[17,18]$ with the strong interaction between Rydberg atoms [19]. Both phenomena have been well studied in the past: It has been demonstrated that photons can be slowed down and stored in atomic gases using EIT [20-22], while recent experiments on Rydberg atoms have demonstrated the strong interaction and the associated blockade of Rydberg excitations [11,23-28]. In the Rydberg-EIT system, a photon entering the atomic gas is converted into a slow-light polariton with a substantial admixture of the Rydberg state. It is the latter admixture that maps the Rydberg-Rydberg interaction onto an effective interaction between slow Rydberg polaritons. Within this approach, a single-photon source [11] and switch [29-31] were realized, the photon blockade [13] and the formation of bound states of Rydberg polaritons [14] have been demonstrated, and atom-photon entanglement was observed [32].

In this paper, we derive the scattering properties and bound-state structure of Rydberg polaritons in one dimension. Our analysis provides a rigorous theoretical framework for analyzing a variety of problems in Rydberg-polariton systems. This framework allows us to analytically derive the effective interaction potential between two Rydberg polaritons and to identify a regime with a purely repulsive interaction. We derive the low-energy scattering length and find the appearance

\footnotetext{
*These two authors contributed equally.

${ }^{\dagger}$ Corresponding author: przemek@theo3.physik.uni-stuttgart.de
}

of resonances; we expect the corresponding tunability of the scattering length to play the role that Feshbach resonances play in ultracold atomic gases [33]. Moreover, we identify multiple two-polariton bound states for attractive interactions and determine their dispersion relation. This understanding paves the way for a microscopic derivation of the many-body theory for Rydberg polaritons in the dilute regime.

Before proceeding we note that first steps towards a fewand many-body theory for Rydberg polaritons in one dimension have already been taken [3]. However, a full description of the system, including the short-range and finite-energy effects relevant to ongoing experiments $[13,14]$, is limited to extended numerical simulations $[10,34]$.

\section{MODEL AND KEY RESULTS}

We consider photons inside an atomic ensemble propagating in one dimension under EIT conditions, where the atomic ground state is coupled to a Rydberg state via an intermediate level referred to as the $p$ state [see Fig. 1(a)]. We introduce the electric-field operators $\psi_{e}^{\dagger}(z)$ and $\psi_{e}(z)$, creating and annihilating a photon at position $z$, respectively. If the atomic density is much higher than the photon density, the excitations of atoms are well described by the bosonic field operators $\psi_{p}^{\dagger}(z)$ and $\psi_{s}^{\dagger}(z)$. Here $\psi_{p}^{\dagger}(z)$ describes the atomic excitation into the $p$ state, while $\psi_{s}^{\dagger}(z)$ generates a Rydberg excitation.

We then obtain the noninteracting part of the microscopic Hamiltonian under the rotating-wave approximation in the rotating frame

$$
H_{0}=\hbar \int d z\left(\begin{array}{l}
\psi_{e} \\
\psi_{p} \\
\psi_{s}
\end{array}\right)^{\dagger}\left(\begin{array}{ccc}
-i c \partial_{z} & g & 0 \\
g & \Delta & \Omega \\
0 & \Omega & 0
\end{array}\right)\left(\begin{array}{l}
\psi_{e} \\
\psi_{p} \\
\psi_{s}
\end{array}\right)
$$

Here $g$ denotes the collective coupling of the photons to the matter via the excitation of ground-state atoms into the $p$ level, while $\Omega$ denotes the Rabi frequency of the control field between the $p$ level and the Rydberg state. Note that the kinetic energy of the photons $-i \hbar \partial_{z}$ only accounts for the deviation from the EIT condition. We introduced the complex detuning $\Delta=\delta-i \gamma$, which accounts for the detuning $\delta$ of the control 

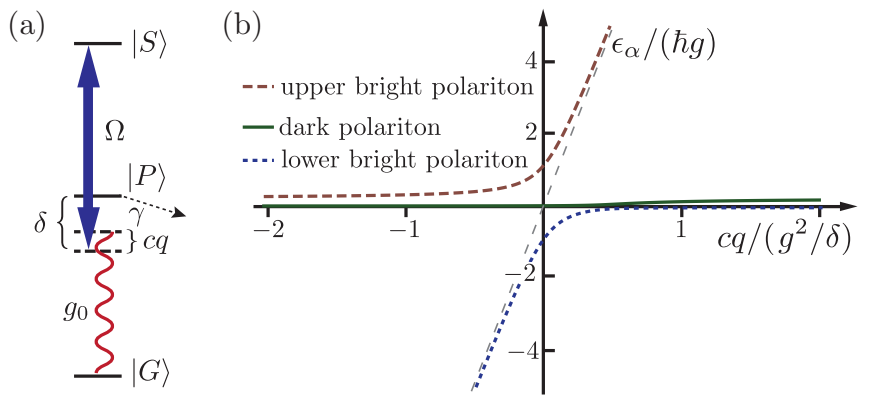

FIG. 1. (Color online) (a) Setup for the electromagnetically induced transparency. The probe field couples the atomic ground state $|G\rangle$ to the $p$ level $|P\rangle$ with the single-particle coupling strength $g_{0}$, while a strong-coupling laser drives the transition between the $p$ level and the Rydberg state $|S\rangle$ with Rabi frequency $\Omega$ and detuning $\delta$. Furthermore, $2 \gamma$ denotes the decay rate from the $p$ level. The single-particle coupling $g_{0}$ is related to the collective coupling $g=\sqrt{n} g_{0}$ with $n$ the particle density. (b) Dispersion relation for the three noninteracting polariton branches for $g=5 \delta, \Omega=0.2 \delta$, and $\gamma=0$.

field and the decay rate $2 \gamma$ from the $p$ level. Throughout our analysis we assume $|\delta| \gg \gamma$, thus providing the results in the limit $\gamma=0$. Then the inclusion of a finite decay rate $\gamma$ is obtained by an analytical continuation in $\Delta=\delta-i \gamma$. This quadratic Hamiltonian is readily diagonalizable in momentum space and we obtain the spectrum shown in Fig. 1(b), which corresponds to two bright polariton modes and one dark polariton. The interaction between the Rydberg levels is described by

$$
H_{\mathrm{rr}}=\frac{1}{2} \int d z d z^{\prime} V\left(z-z^{\prime}\right) \psi_{s}^{\dagger}(z) \psi_{s}^{\dagger}\left(z^{\prime}\right) \psi_{s}\left(z^{\prime}\right) \psi_{s}(z)
$$

In the following, we focus on a van der Waals interaction $V(r)=C_{6} / r^{6}$. The microscopic Hamiltonian $H_{0}+H_{\mathrm{rr}}$ describes three bosonic fields with a noninteracting quadratic part and a quartic interaction. However, the quadratic Hamiltonian exhibits a rather unconventional form, as the only dynamics is given by the light velocity of the photon. It is this property, together with the conservation of total energy $\hbar \omega$ and centerof-mass momentum $\hbar K$, that is crucial to our analysis and gives rise to different phenomena.

In order to understand the many-body properties of the system, we will analyze the scattering properties and boundstate structure for two polaritons. The main idea is to derive the scattering length $a_{1 \mathrm{D}}$, which in turn allows for the description of the many-body theory in terms of a pseudopotential. In one dimension, the pseudopotential takes the form [35]

$$
V_{1 \mathrm{D}}(r)=-\frac{2 \hbar^{2}}{m a_{1 \mathrm{D}}} \delta(r),
$$

where $m$ plays the role of the polariton mass (see below). This approach is analogous to those used in describing cold atomic gases, where it is extremely successful [33].

The description of two polaritons requires, in the most general case, a nine-component, two-particle wave function $\psi_{\mu \nu}\left(z, z^{\prime}\right)$ with $\mu, v \in\{e, p, s\}$, which denotes the amplitude of finding particles in states $\mu$ and $v$ at $z$ and $z^{\prime}$, respectively [10]. To utilize the conservation of energy and momentum, we rewrite $\psi_{\mu \nu}$ in the center of mass $R=\left(z+z^{\prime}\right) / 2$ and relative $r=z-z^{\prime}$ coordinates and parametrize it in terms of temporal and spatial Fourier components $(\omega, K)$, leaving $r$ the only degree of freedom.

In the following, we first present the main results and discuss its derivation later. We find that the quantum dynamics of the two polaritons is well captured by a Schrödinger-like equation for a single component

$$
\hbar \bar{\omega} \psi(r)=\left[-\frac{\hbar^{2}}{m} \partial_{r}^{2}+\alpha V^{\mathrm{eff}}(r)\right] \psi(r),
$$

with the effective potential and polariton mass

$$
V^{\mathrm{eff}}(r)=\frac{V(r)}{1-\bar{\chi}(\omega) V(r)}, \quad m=\hbar \frac{\left(g^{2}+\Omega^{2}\right)^{3}}{2 c^{2} g^{2} \Delta \Omega^{2}} .
$$

Here $\hbar \bar{\omega}(K, \omega)$ plays the role of energy and the dimensionless parameter $\alpha(K, \omega)$ can be interpreted as the overlap of the polaritons with the Rydberg state. The effective interaction potential is renormalized as the interaction shifts the two Rydberg states out of resonance and its saturation value as two polaritons approach each other is defined as $-1 / \bar{\chi}(\omega)$. The wave function $\psi(r)$ is related to the two-body wave functions via $\psi(r)=\psi_{s s}(r)[1-\bar{\chi} V(r)]$. Note that this relation captures the blockade phenomenon: The amplitude $\psi_{s s}(r)$ to find two Rydberg states essentially vanishes at distances shorter than the blockade radius $\xi=\left(\left|C_{6} \bar{\chi}\right|\right)^{1 / 6}$. In addition, the wave function $\psi(r)$ is proportional to the electric-field amplitude $\psi_{e e}(r)$. Equation (4) is valid in several relevant regimes, including the low-momentum and low-energy regime and the far-detuned regime; the analytical expressions for $\alpha, \bar{\omega}$, and $\bar{\chi}$ are discussed below.

We start with the low-momentum and low-energy regime, which allows us to analytically derive the low-energy scattering length $a_{1 \mathrm{D}}$. When $|\omega|,|K| v_{g} \ll \min \left\{|\Delta|, 2 \Omega^{2} /|\Delta|\right\}$, where $v_{g}=\Omega^{2} /\left(\Omega^{2}+g^{2}\right) c$ is the slow-light velocity, the expressions for $\bar{\omega}$ and $\alpha$ are in leading order

$$
\bar{\omega}=\omega-v_{g} K, \quad \alpha=\frac{g^{4}}{\left(g^{2}+\Omega^{2}\right)^{2}} .
$$

In this limit, Eq. (4) provides the intuitive result: $\hbar \bar{\omega}$ is the difference between the total energy $\hbar \omega$ and the kinetic energy $\hbar v_{g} K$ of the center-of-mass motion and $\alpha$ is the square of the probability of finding a polariton in the Rydberg state. Furthermore, the amplitude for the electric field takes the form $\psi_{e e}=\Omega^{2} / g^{2} \psi$. At the same time, $\bar{\chi}$ reduces to

$$
\hbar \bar{\chi}=\frac{\Delta}{2 \Omega^{2}}-\frac{1}{2 \Delta},
$$

which exhibits a zero crossing for $\Omega= \pm \Delta$ with an associated sign change. Therefore, it is possible to realize effectively repulsive polariton-polariton interactions for $\Omega>|\Delta|$; note that the character of the interaction is determined by the ratio of its saturation and the polariton mass $\bar{\chi} / \mathrm{m}$. It is in contrast to the far-off-resonance regime $(\Omega \ll|\Delta|)$, where the combination of polariton mass and effective interaction always leads to an effective attraction [14]. In this regime, we observe a transition from a negative to a positive one-dimensional scattering length $a_{1 \mathrm{D}}$ for increasing interactions [see Fig. 2(a)]. The interaction strength is conveniently expressed by the dimensionless 




FIG. 2. (Color online) (a) Low-energy scattering length $a_{1 \mathrm{D}}$. For attractive interactions (solid line), we obtain scattering resonances associated with the appearance of additional bound states for increasing interaction strength. The latter is represented by the so-called blockade radius $\xi$. The normalization $\lambda$ is defined in the text. For repulsive interactions (dashed line), we find a single zero crossing. (b) Two-polariton spectrum for $\Omega \ll|\Delta| \lesssim g$. For weak interactions $\xi / \lambda=0.5$ (dashed line), we obtain a single bound state below the continuum of scattering states, whereas for strong interactions $\xi / \lambda=5$ (solid lines), we observe the existence of several bound states.

parameter $\xi / \lambda$ with the blockade radius $\xi=\left(\left|C_{6} \bar{\chi}\right|\right)^{1 / 6}$ and $\lambda=\sqrt{\left|\hbar^{2} \bar{\chi} / \alpha m\right|}$ the de Broglie wavelength associated with the depth and height of the effective potential. Then we obtain the asymptotic behavior $a_{1 \mathrm{D}}=-(3 / \pi) \lambda^{2} / \xi$, valid for weak interactions with $\xi / \lambda \ll 1$, where the interaction potential can be replaced by a $\delta$ function. Note that for $\gamma=0$, the scattering length is negative, while for a finite decay rate $\gamma>0$, the analytical continuation of the scattering length reduces to $a_{1 \mathrm{D}}=-(3 / \pi)\left(-\bar{\chi}^{5} / C_{6}\right)^{1 / 6}\left(\hbar^{2} / \alpha m\right)$ and gives rise to an imaginary contribution accounting for losses from the $p$ level during the collision. For increasing interactions, eventually a zero crossing of $a_{1 \mathrm{D}}$ appears and we obtain the positive scattering length $a_{1 \mathrm{D}} \approx 0.7\left(\alpha m C_{6} / \hbar^{2}\right)^{1 / 4}$, where the full tail of the van der Waals interaction dominates.

In the attractive regime $\Omega<|\Delta|$ with $C_{6} \delta<0$, the system generally gives rise to bound states. Note that bound states can be identified by negative values of $\bar{\omega}$. For weak interactions $\xi<\lambda$, a single bound state is present and we recover the expression for the scattering length $a_{1 \mathrm{D}}=(3 / \pi) \lambda^{2} / \xi$, which is now positive. For increasing interactions $\xi>\lambda$, additional bound states will appear. Each additional bound state is associated with a resonance in the scattering length in analogy to Feshbach resonances in cold atomic gases [33]. The exact determination of the scattering length $a_{1 \mathrm{D}}$ requires the full treatment of the effective interaction potential $V^{\text {eff }}(r)$; the latter is easily achieved numerically [see Fig. 2(a)]. It clearly demonstrates that we can tune the scattering length to arbitrary values by controlling the single parameter $\xi / \lambda$, which defines the strength of the interaction potential.

In general, the bound states will violate the condition of low energy and are thus more appropriately studied next in the far-detuned regime with $\Omega \ll|\Delta|$, which is valid for all momenta $\hbar K$ with the weak constraint $|\omega| \ll|\Delta|$. In this regime, we obtain $\hbar \bar{\chi}(\omega)=\left(\omega+2 \Omega^{2} / \Delta\right)^{-1}$ and the blockade radius reduces to $\xi=\left(\left|C_{6} \Delta / 2 \Omega^{2} \hbar\right|\right)^{1 / 6}$; the analytic but lengthy expressions for $\bar{\omega}$ and $\alpha$ are presented in the Appendix. In the experimentally most interesting regime of slow light $g \gg \Omega$ with $g \gtrsim|\Delta|$, we find

$$
\alpha=\frac{1-\frac{c K \Delta}{2 g^{2}}}{\left(1+\frac{\omega \Delta}{2 \Omega^{2}}\right)^{2}},
$$

while the expression for the energy $\hbar \bar{\omega}$ is defined through

$$
\frac{\bar{\omega} \Delta}{2 \Omega^{2}}=\frac{\frac{\omega \Delta}{2 \Omega^{2}}}{1+\frac{\omega \Delta}{2 \Omega^{2}}}-\frac{1+2 \frac{\omega \Delta}{2 \Omega^{2}}}{1+\frac{\omega \Delta}{2 \Omega^{2}}} \frac{c K \Delta}{2 g^{2}}+\left(\frac{c K \Delta}{2 g^{2}}\right)^{2} .
$$

Finally, the relation to the electric-field amplitude $\psi_{e e}$ is again closely related to the wave function $\psi$ via $\left(g^{2}-\right.$ $c K \Delta / 2) \psi_{e e}=\left(\Omega^{2}+\omega \Delta / 2\right) \psi$. It is important to stress that, in this limit, our result agrees with the approach utilizing adiabatic elimination (see the Appendix).

The effective equation (4) allows us to derive the bound states and their group velocity in addition to the scattering states. The spectrum is shown in Fig. 2(b): It exhibits a continuum of scattering states as well as bound states. Note that the interaction potential and $\bar{\omega}$ depend on the energy $\hbar \omega$ and therefore the bound-state energies have to be determined self-consistently. The dimensionless parameter measuring the strength of the interaction reduces to $\xi / \lambda=\xi g^{2} /|\Delta| c$. Then, for weak interactions $\xi / \lambda<1$, we recover a single bound state, which is well described by replacing the effective interaction potential by a $\delta$ function. For increasing interaction strength $\xi / \lambda>1$, we observe the appearance of additional bound states. Then the exact bound-state energy requires the numerical treatment of the full effective interaction potential (5). The result is shown in Fig. 2(b) for two different interaction strengths.

\section{DERIVATION AND LIMITATIONS}

Next we present the microscopic derivation of Eq. (4) and discuss its limitations. We start by diagonalizing the noninteracting part of the Hamiltonian in momentum space obtaining the dispersion relations of three polariton modes [shown in Fig. 1(b)] $H_{0}=\sum_{q, \mu \in 0, \pm 1} \epsilon_{\mu q} \bar{\psi}_{\mu q}^{\dagger} \tilde{\psi}_{\mu q}$. Here $\tilde{\psi}_{ \pm 1 q}$ account for the two bright polariton modes, while $\tilde{\psi}_{0 q}$ denotes the dark polariton. The new field operators take the form $\tilde{\psi}_{\mu q}=\sum_{v \in\{e, p, s\}} U_{\mu}^{v}(q) \psi_{v q}$ with $\mu \in\{0, \pm 1\}$ and the inverse $\bar{U} \equiv U^{-1}$ provides $\bar{\psi}_{\mu q}^{\dagger}=\sum_{v \in\{e, p, s\}} \bar{U}_{\mu}^{v}(q) \psi_{\nu q}^{\dagger}$.

The two-polariton scattering properties are well accounted for by the $T$ matrix. As the interaction acts only between the two Rydberg states, it is sufficient to study the $T$ matrix for the Rydberg states alone, denoted by $T_{k k^{\prime}}(K, \omega)$. Here $\hbar k$ is the relative momentum of the two incoming polaritons and $\hbar k^{\prime}$ the relative momentum of the outgoing polaritons. For two polaritons, the $T$ matrix is determined by the integral equation [36]

$$
T_{k k^{\prime}}(K, \omega)=V_{k-k^{\prime}}+\int \frac{d q}{2 \pi} V_{k-q} \chi_{q}(K, \omega) T_{q k^{\prime}}(K, \omega),
$$

which can easily be understood as a resummation of all ladder diagrams [see Fig. 3(a)]. The full pair propagator of two polaritons and its overlap with the Rydberg state takes the 

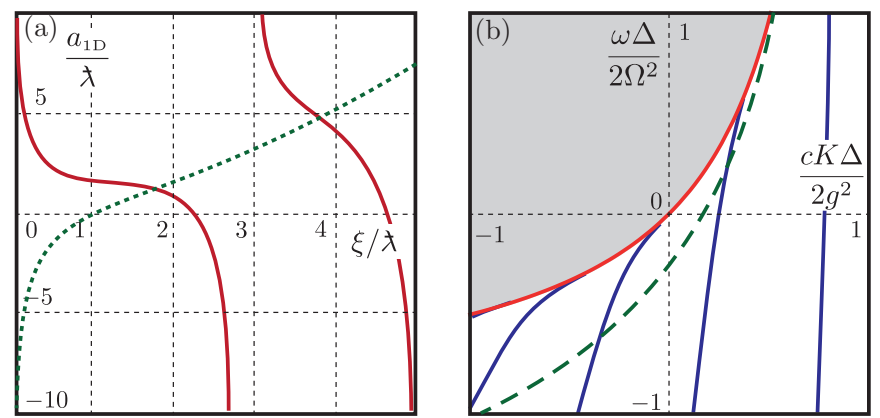

FIG. 3. (Color online) (a) Illustration of ladder diagrams up to fourth order. The interaction $V(r)$ is denoted by a wavy line, while the straight lines with an arrow are Green's functions for the three polariton modes $1 /\left(\hbar \omega-\epsilon_{\mu}+i \eta\right)$ and the dots mark the overlap factors $U_{\mu}^{s}$ and $\bar{U}_{s}^{\mu}$ of the polariton with the Rydberg state. The $T$ matrix includes all diagrams to arbitrary order with all possible intermediate polaritons. (b) Parameter $\zeta(K, \omega)$ measuring the influence of the second pole for $g \gg \Omega$ and $\Omega / \Delta=0.5$. In the lowenergy, low-momentum limit, the second pole can be safely neglected, however its influence strongly increases for $K c \Delta / 2 g^{2} \sim 1$.

form

$$
\chi_{q}(K, \omega)=\sum_{\alpha, \beta \in\{0, \pm 1\}} \frac{\bar{U}_{s}^{\alpha}(p) U_{\alpha}^{s}(p) \bar{U}_{s}^{\beta}\left(p^{\prime}\right) U_{\beta}^{s}\left(p^{\prime}\right)}{\hbar \omega-\epsilon_{\alpha}(p)-\epsilon_{\beta}\left(p^{\prime}\right)+i \eta},
$$

with $p=K / 2+q$ and $p^{\prime}=K / 2-q$. It is a special property of our polariton Hamiltonian that the pair propagation reduces to three terms

$$
\chi_{q}=\bar{\chi}+\frac{\alpha}{\hbar \bar{\omega}-\hbar^{2} q^{2} / m+i \eta}+\frac{\alpha_{B}}{\hbar \bar{\omega}_{B}-\hbar^{2} q^{2} / m+i \eta} .
$$

Here $\bar{\chi}(\omega)$ accounts for the saturation of the pair propagation at large momenta $\hbar q \rightarrow \pm \infty$ and takes the form

$$
\bar{\chi}(\omega)=\frac{1}{\hbar} \frac{\Delta-\frac{\omega}{2}-\frac{\Omega^{2}}{\Delta-\omega}}{\omega\left(\Delta-\frac{\omega}{2}\right)+2 \Omega^{2}} .
$$

The second term in Eq. (12) is the pole structure for the propagation of the two incoming polaritons. This term reduces to the propagator of a single massive particle, where $\alpha$ and $\bar{\omega}$ depend on the center-of-mass momentum $\hbar K$ and total energy $\hbar \omega$. The latter defines the relative momentum $\hbar k= \pm \sqrt{m \bar{\omega}} / \hbar$ of the incoming scattering states. Finally, the last term accounts for a second pole, describing the phenomenon of resonant scattering of two incoming polaritons into a different outgoing channel, e.g., the conversion of two dark polaritons into an upper and a lower bright polariton, and therefore is denoted by $B$. The influence of the second pole is measured by the dimensionless parameter $\zeta(K, \omega)=\sqrt{\left|\bar{\omega} \alpha_{B}^{2} / \bar{\omega}_{B} \alpha^{2}\right|}$. In particular, $\zeta(K, \omega)$ is strongly suppressed in the two regimes discussed above; an illustration demonstrating the strong suppression is shown Fig. 3(b), while the analytical expressions are provided in the Appendix. In these cases the second pole can be dropped in leading order in the small parameter $\zeta \ll 1$.

The saturation $\bar{\chi}$ can be eliminated by introducing the effective interaction potential $V^{\text {eff }}(r)$ given in Eq. (5). Then the equation for the $T$ matrix reduces to

$$
T_{k k^{\prime}}=V_{k-k^{\prime}}^{\mathrm{eff}}+\int \frac{d q}{2 \pi} V_{k-q}^{\mathrm{eff}} \frac{\alpha}{\hbar \bar{\omega}-\hbar^{2} q^{2} / m} T_{q k^{\prime}} .
$$

Consequently, the $T$ matrix describes a system of a single massive particle in the effective interaction potential $V_{\text {eff }}$ with the relative coordinate as the degree of freedom and is fully described by the Schrödinger equation (4). The relation $\psi(r)=\psi_{s s}(r)[1-\bar{\chi} V(r)]$ follows from the relation between the $T$ matrix and the scattering wave function $\psi_{s s}(r) V(r)=$ $\int d k^{\prime} e^{i r k^{\prime}} T_{k k^{\prime}} /(2 \pi)=\psi(r) V^{\text {eff }}(r)$.

\section{MANY-BODY HAMILTONIAN}

The full understanding of the scattering properties allows us to derive the low-energy many-body Hamiltonian for Rydberg polaritons. Here the fundamental assumption is that each scattering process of the polaritons is independent of each other. This condition is satisfied in the dilute regime $n_{d} r_{0} \ll 1$, where the density $n_{d}$ of Rydberg polaritons is low compared to the range $r_{0}$ of the interaction potential. The latter is determined by either the blockade radius or the van der Waals length, i.e., $r_{0}=\max \left\{\xi,\left(\left|\alpha m C_{6}\right| / \hbar^{2}\right)^{1 / 4}\right\}$. Then the interaction is fully determined by the scattering length $a_{1 \mathrm{D}}$ via the one-dimensional pseudopotential $V_{1 \mathrm{D}}=-2 \hbar^{2} / m a_{1 \mathrm{D}} \delta(r)$ [35] and the many-body theory reduces to the Hamiltonian

$H=\int d x\left[\psi_{d}^{\dagger}\left(-i \hbar v_{g} \partial_{z}-\frac{\hbar^{2}}{2 m} \partial_{z}^{2}\right) \psi_{d}-\frac{2 \hbar^{2}}{m a_{1 \mathrm{D}}} \psi_{d}^{\dagger} \psi_{d}^{\dagger} \psi_{d} \psi_{d}\right]$,

with $\psi_{d}^{\dagger}\left(\psi_{d}\right)$ denoting the bosonic field operator creating (annihilating) a Rydberg polariton. Here we can control the scattering length $a_{1 \mathrm{D}}$ by the strength of the interactions [see Fig. 2(a)]. We can therefore study continuously the crossover from a Lieb-Liniger gas at $a_{1 \mathrm{D}}<0$ to the super Tonks-Girardeaux gas at $a_{1 \mathrm{D}}>0$ by tuning the parameters through a zero crossing of the scattering length [37-39]. In contrast to cold atomic gases, here we do not have a competing resonance-inducing losses [40,41].

Finally, we point out that a complementary derivation of an effective low-energy theory can also be achieved at high densities if the interaction is dominated by the purely repulsive part of the van der Waals interaction, as proposed in Ref. [3]. This theory is of interest in the low-momentum regime with $1 /\left(\left|\alpha m C_{6}\right| / \hbar^{2}\right)^{1 / 4}<n_{1 \mathrm{D}}<1 /|\xi|$; note that here we provide a microscopic derivation for the short-distance behavior. We have demonstrated that this regime is most interesting to study when $\Delta \approx \pm \Omega$ and $C_{6} \delta>0$, where $\bar{\chi}$ is strongly suppressed and the effective interaction reduces to the pure van der Waals repulsion $V^{\mathrm{eff}}(r)=C_{6} / r^{6}$. This scenario allows one to observe the crossover into a regime where crystalline correlations dominate the ground state.

\section{OUTLOOK}

The microscopic analysis presented here has several implications for experiments. First, the existence of a parameter regime with a purely repulsive interaction will give rise to photon antibunching for the two-photon correlations in 
an experimental setup similar to that of Ref. [14]. The experimental requirements are a strong Rabi frequency $\Omega \gtrsim$ $|\Delta|$ and $\gamma \ll|\delta|$ to distinguish the repulsion from losses. In turn, the analysis of the bound-state structure allows for the determination of the group velocity. As can be seen in Fig. 2(b), the group velocity of the bound states is larger than the slow-light velocity and the bound states will travel ahead of the continuum. This will allow one to spatially separate the bound photon pairs in a pulsed experiment. Finally, the scattering length defines the phase shift two polaritons pick up during a collision; it has been proposed to use such collisions to realize photonic two-qubit gates $[8,10]$. Here the predicted zero crossing of the scattering length corresponds to the optimal $\pi$-phase shift. A direct measurement of these resonances is possible in a setup with frequency difference $\Delta \omega$ and spatially resolved detection of the polaritons inside the medium. Therein, the correlation function in the relative coordinate will oscillate with a wave vector $\Delta k=\Delta \omega / v_{g}$. The maxima of these oscillations will shift for increasing scattering length by a phase $\phi$ via $\cot (\phi)=-a_{1 \mathrm{D}} \Delta k$. The details of these observations depend on the experimental setup and on the precise boundary conditions but can be efficiently addressed within the presented framework.

\section{ACKNOWLEDGMENTS}

We thank T. Pohl, M. Fleischhauer, P. Strack, and M. Hafezi for discussions. We acknowledge support from the Center for Integrated Quantum Science and Technology, the Deutsche Forschungsgemeinschaft within SFB TRR 21, the EU Marie Curie ITN COHERENCE, CUA, AFOSR MURI, DARPA QUINESS, Packard Foundation, the JQI, the NSF PFC at the JQI, NSF PIF, the U.S. Army Research Laboratory (ARL), the Army Research Office (ARO), and the National Science Foundation. H.P.B., M.D.L., and A.V.G. acknowledge hospitality of the KITP.

\section{APPENDIX}

\section{Influence and strength of the second pole}

In the following, we estimate the relevance of the second pole in Eq. (10), characterized by $\alpha_{B}$ and $\bar{\omega}_{B}$, which gives rise to the resonant scattering into a different outgoing channel. First, we concentrate on the low-momentum and -energy regime. The analytical expressions for $\alpha$ and $\bar{\omega}$ describing the first pole are given by Eq. (5). In turn, the parameters for the second pole derived by the diagrammatic method take the form

$$
\begin{gathered}
\alpha_{B}=-\frac{(\omega-c K)^{2} \Omega^{6}}{4 \Delta^{2}\left(g^{2}+\Omega^{2}\right)^{3}}, \\
\bar{\omega}_{B}=\frac{4 \Omega^{2} g^{4}}{\left(g^{2}+\Omega^{2}\right)^{3}} \frac{\Delta^{2}}{\omega-c K} .
\end{gathered}
$$

Note that the weight $\alpha_{B}$ of the pole vanishes quadratically when $\hbar|\omega|, \hbar|K| v_{g} \ll \hbar \min \left\{|\Delta|, 2 \Omega^{2} /|\Delta|\right\}$, where $v_{g}=$ $\Omega^{2} /\left(\Omega^{2}+g^{2}\right) c$ is the slow-light velocity, and can therefore be safely dropped.

Next we analyze the influence of the second pole in the regime of far-detuned Rydberg polaritons with $|\omega|, \Omega \ll$
$|\Delta|, g$. The diagrammatic approach provides the analytic expressions

$$
\begin{aligned}
\alpha_{B} & =-\frac{\Omega^{6}\left(1+\frac{c K}{2 \Delta}\right)(\omega-c K)^{2}}{4 \Delta^{2}\left(g^{2}+\Omega^{2}\right)^{3}\left(1-\frac{c K \Delta}{2 g^{2}}\right)^{2}} \\
\bar{\omega}_{B} & =-\left(1+\frac{c K}{2 \Delta}\right)^{2}\left(1-\frac{c K \Delta}{2 g^{2}}\right) \frac{4 \Omega^{2} g^{4}}{\left(g^{2}+\Omega^{2}\right)^{3}} \frac{\Delta^{2}}{c K-\omega} .
\end{aligned}
$$

We find that, in the regime $c K \delta / 2 g^{2}<1$, the dimensionless parameter $\zeta(K, \omega)$ is strongly suppressed by the factor $(\Omega / \Delta)^{6}$. However, it is important to stress that the strength of the second pole diverges in a narrow parameter regime around $c K \delta / 2 g^{2} \approx 1$.

\section{Adiabatic elimination}

In the following, we compare our diagrammatic approach with the previous successfully applied study of the twoparticle equation for the wave function in the regime $\omega=$ 0 , where the $p$ level has sometimes been adiabatically eliminated [10,13,14]. Furthermore, we present the natural extension of adiabatic elimination for finite frequencies. Then the two-particle wave function contains four components: $\psi_{e e}$ describes the amplitude for two photons, $\psi_{s s}$ the amplitude for two Rydberg atoms, and $\psi_{e s \pm}$ the amplitude for one photon and one Rydberg atom with even (odd) symmetry. The Schrödinger equation reduces to (see Refs. [10,13,14] for more details)

$$
\begin{array}{r}
\omega \psi_{e e}=-i c \partial_{R} \psi_{e e}-\frac{2 g^{2}}{\Delta} \psi_{e e}-\frac{2 g \Omega}{\Delta} \psi_{e s+}, \\
\omega \psi_{e s+}=-\frac{i c}{2} \partial_{R} \psi_{e s+}-i c \partial_{r} \psi_{e s-} \\
\quad-\frac{g^{2}+\Omega^{2}}{\Delta} \psi_{e s+}-\frac{g \Omega}{\Delta}\left(\psi_{e e}+\psi_{s s}\right), \\
\omega \psi_{e s-}=-\frac{i c}{2} \partial_{R} \psi_{e s-}-i c \partial_{r} \psi_{e s+}-\frac{g^{2}+\Omega^{2}}{\Delta} \psi_{e s-}, \\
\omega \psi_{s s}=-\frac{2 \Omega^{2}}{\Delta} \psi_{s s}-\frac{2 g \Omega}{\Delta} \psi_{e s+}+\frac{V(r)}{\hbar} \psi_{s s},
\end{array}
$$

where $r$ denotes the relative coordinate and $R$ the center-ofmass coordinate. For the translational invariant system, the latter coordinate is expressed in Fourier space with $K$ the total momentum. We can solve Eqs. (A4), (A6), and (A7) for $\psi_{e e}$, $\psi_{e s-}$, and $\psi_{s s}$, respectively.

Inserting these expressions into Eq. (A5), we obtain a single-differential equation involving only the wave function $\psi_{\text {es+ }}$,

$$
\hbar \bar{\omega} \psi_{e s+}=-\frac{\hbar^{2}}{m} \partial_{r}^{2} \psi_{e s+}+\alpha V_{\text {eff }}(r) \psi_{e s+} .
$$

This equation takes exactly the form of Eq. (3) with the identification $\psi_{e s+} \sim \psi$. The expressions for $\alpha$ and $\bar{\omega}$ within the adiabatic elimination reduce to

$$
\frac{\alpha m}{\hbar^{2}}=\frac{g^{2} \Omega^{2}}{c^{2} \hbar \Delta^{2}} \frac{2\left(\omega+\frac{g^{2}+\Omega^{2}}{\Delta}\right)-c K}{\left(\omega+2 \frac{\Omega^{2}}{\Delta}\right)^{2}},
$$




$$
\begin{aligned}
\bar{\chi} & =\frac{1}{\hbar} \frac{1}{\omega+2 \Omega^{2} / \Delta}, \\
\frac{\bar{\omega} m}{\hbar} & =\left[c K-2\left(\omega+\frac{\Omega^{2}+g^{2}}{\Delta}\right)\right]^{2} \frac{2 \omega \frac{\Omega^{2}+g^{2}}{\Delta}+\omega(\omega-c K)-\frac{2 \Omega^{2}}{\Delta} c K}{4 c^{2}\left(\omega+\frac{2 \Omega^{2}}{\Delta}\right)\left(\omega-c K+\frac{2 g^{2}}{\Delta}\right)} .
\end{aligned}
$$

These expressions fully agree with the result derived within the diagrammatic approach in the limit of large detuning $\Omega \ll|\Delta|$ and energies $|\omega| \ll|\Delta|$. In the physically interesting situation of Rydberg polaritons with $g \gtrsim|\Delta|$, we finally obtain the expressions (7) and (8).

[1] I. Carusotto and C. Ciuti, Rev. Mod. Phys. 85, 299 (2013).

[2] D. E. Chang, V. Gritsev, G. Morigi, V. Vuletic, M. D. Lukin, and E. A. Demler, Nat. Phys. 4, 884 (2008).

[3] J. Otterbach, M. Moos, D. Muth, and M. Fleischhauer, Phys. Rev. Lett. 111, 113001 (2013).

[4] I. Fushman, D. Englund, A. Faraon, N. Stoltz, P. Petroff, and J. Vuckovic, Science 320, 769 (2008).

[5] A. Rauschenbeutel, G. Nogues, S. Osnaghi, P. Bertet, M. Brune, J. M. Raimond, and S. Haroche, Phys. Rev. Lett. 83, 5166 (1999).

[6] G. Kirchmair, B. Vlastakis, Z. Leghtas, S. E. Nigg, H. Paik, E. Ginossar, M. Mirrahimi, L. Frunzio, S. M. Girvin, and R. J. Schoelkopf, Nature (London) 495, 205 (2013).

[7] M. J. Hartmann, F. G. S. L. Brandao, and M. B. Plenio, Laser Photon. Rev. 2, 527 (2008).

[8] I. Friedler, D. Petrosyan, M. Fleischhauer, and G. Kurizki, Phys. Rev. A 72, 043803 (2005).

[9] J. D. Pritchard, D. Maxwell, A. Gauguet, K. J. Weatherill, M. P. A. Jones, and C. S. Adams, Phys. Rev. Lett. 105, 193603 (2010).

[10] A. V. Gorshkov, J. Otterbach, M. Fleischhauer, T. Pohl, and M. D. Lukin, Phys. Rev. Lett. 107, 133602 (2011).

[11] Y. O. Dudin and A. Kuzmich, Science 336, 887 (2012).

[12] V. Parigi, E. Bimbard, J. Stanojevic, A. J. Hilliard, F. Nogrette, R. Tualle-Brouri, A. Ourjoumtsev, and P. Grangier, Phys. Rev. Lett. 109, 233602 (2012).

[13] T. Peyronel, O. Firstenberg, Q.-Y. Liang, S. Hofferberth, A. V. Gorshkov, T. Pohl, M. D. Lukin, and V. Vuletić, Nature (London) 488, 57 (2012).

[14] O. Firstenberg, T. Peyronel, Q.-Y. Liang, A. V. Gorshkov, M. D. Lukin, and V. Vuletić, Nature (London) 502, 71 (2013).

[15] D. Petrosyan, J. Otterbach, and M. Fleischhauer, Phys. Rev. Lett. 107, 213601 (2011).

[16] M. D. Lukin, M. Fleischhauer, R. Coté, L. M. Duan, D. Jaksch, J. I. Cirac, and P. Zoller, Phys. Rev. Lett. 87, 037901 (2001).

[17] M. Fleischhauer and M. D. Lukin, Phys. Rev. Lett. 84, 5094 (2000).

[18] M. Fleischhauer, A. Imamoglu, and J. P. Marangos, Rev. Mod. Phys. 77, 633 (2005).

[19] M. Saffman, T. Walker, and K. Mølmer, Rev. Mod. Phys. 82, 2313 (2010).

[20] C. Liu, Z. Dutton, C. H. Behroozi, and L. V. Hau, Nature (London) 409, 490 (2001).

[21] D. F. Phillips, A. Fleischhauer, A. Mair, R. L. Walsworth, and M. D. Lukin, Phys. Rev. Lett. 86, 783 (2001).

[22] B. Julsgaard, J. Sherson, J. I. Cirac, J. Fiurasek, and E. S. Polzik, Nature (London) 432, 482 (2004).
[23] A. Gaëtan, Y. Miroshnychenko, T. Wilk, A. Chotia, M. Viteau, D. Comparat, P. Pillet, A. Browaeys, and P. Grangier, Nat. Phys. 5, 115 (2009).

[24] E. Urban, T. A. Johnson, T. Henage, L. Isenhower, D. D. Yavuz, T. G. Walker, and M. Saffman, Nat. Phys. 5, 110 (2009).

[25] H. Schempp, G. Günter, M. Robert-de-Saint-Vincent, C. S. Hofmann, D. Breyel, A. Komnik, D. W. Schönleber, M. Gärttner, J. Evers, S. Whitlock, and M. Weidemüller, Phys. Rev. Lett. 112, 013002 (2014).

[26] D. Maxwell, D. J. Szwer, D. Paredes-Barato, H. Busche, J. D. Pritchard, A. Gauguet, K. J. Weatherill, M. P. A. Jones, and C. S. Adams, Phys. Rev. Lett. 110, 103001 (2013).

[27] P. Schausz, M. Cheneau, M. Endres, T. Fukuhara, S. Hild, A. Omran, T. Pohl, C. Gross, S. Kuhr, and I. Bloch, Nature (London) 491, 87 (2012).

[28] R. Heidemann, U. Raitzsch, V. Bendkowsky, B. Butscher, R. Löw, L. Santos, and T. Pfau, Phys. Rev. Lett. 99, 163601 (2007).

[29] H. Gorniaczyk, C. Tresp, J. Schmidt, H. Fedder, and S. Hofferberth, Phys. Rev. Lett. 113, 053601 (2014).

[30] S. Baur, D. Tiarks, G. Rempe, and S. Dürr, Phys. Rev. Lett. 112, 073901 (2014).

[31] D. Tiarks, S. Baur, K. Schneider, S. Dürr, and G. Rempe, Phys. Rev. Lett. 113, 053602 (2014).

[32] L. Li, Y. O. Dudin, and A. Kuzmich, Nature (London) 498, 466 (2013).

[33] C. Chin, R. Grimm, P. Julienne, and E. Tiesinga, Rev. Mod. Phys. 82, 1225 (2010).

[34] B. He, A. V. Sharypov, J. Sheng, C. Simon, and M. Xiao, Phys. Rev. Lett. 112, 133606 (2014).

[35] M. Olshanii, Phys. Rev. Lett. 81, 938 (1998).

[36] A. Abrikosov, L. Gorkov, and I. Dzyaloshinski, Methods of Quantum Field Theory in Statistical Physics (Dover, New York, 1963).

[37] G. E. Astrakharchik, J. Boronat, J. Casulleras, and S. Giorgini, Phys. Rev. Lett. 95, 190407 (2005).

[38] S. Chen, L. Guan, X. Yin, Y. Hao, and X.-W. Guan, Phys. Rev. A 81, 031609 (2010).

[39] M. D. Girardeau and G. E. Astrakharchik, Phys. Rev. A 81, 061601 (2010).

[40] E. Haller, M. Gustavsson, M. J. Mark, J. G. Danzl, R. Hart, G. Pupillo, and H.-C. Nägerl, Science 325, 1224 (2009).

[41] S. Sala, P.-I. Schneider, and A. Saenz, Phys. Rev. Lett. 109, 073201 (2012). 